\title{
CANCER
}

\section{Double death for cancer cells}

DOI:

10.1038/nrd2273
Tumour cells that are treated with chemotherapy or radiation frequently initiate autophagy, a lysosomedependent intracellular degradation pathway. However, it is controversial whether this allows tumour cells to survive therapy, or whether it represents the initiation of a non-apoptotic form of cell death. Reporting in the Journal of Clinical Investigation, Amaravadi and colleagues show that blocking autophagy in addition to chemotherapy leads to accelerated cancer cell death. This provides a rationale for further investigation of autophagy inhibitors as potentiators of anticancer agents.

In their study, the authors used a model system of B-cell lymphoma in which $M y c$-induced tumour cells generated in p53-oestrogen receptor knock-in mice ( $\mathrm{p} 53 \mathrm{ER}^{\mathrm{TAM}}$ ) were adoptively transferred to syngeneic mice. In these mice, the function of the tumour suppressor p53 can be temporally regulated by giving the drug tamoxifen, which allows the p53-oestrogen-receptor fusion protein to translocate to the nucleus and restore p53 function. Activation of p53 led to apoptosis induction and tumour regression. However, all animals experienced tumour recurrence despite continuous treatment with tamoxifen. Additional treatment with chloroquine, which, at low doses, inhibits autophagy, resulted in a delay in tumour recurrence. Importantly, when treated simultaneously with chloroquine and tamoxifen, $81 \%$ of mice experienced a complete clinical regression of the tumour, compared with $8 \%$ of mice treated with tamoxifen alone. Molecular and electron microscopy analysis revealed that this was a direct consequence of p53-dependent apoptosis enhancement that was due to the inhibition of autophagy-based survival mechanisms by chloroquine. To confirm these results, the authors used RNA interference (short hairpin RNA) targeted at the autophagy gene ATG5, which generated comparable results to those of chloroquine treatment.

As alkylating agents such as cyclophosphamide are usually first-line treatments for human lymphomas, mice with Myc/p53ER ${ }^{\text {TAM }}$ lymphomas were treated with cyclophosphamide instead of tamoxifen, alone or in combination with chloroquine. After 24 hours of treatment, the size of tumours in mice receiving the drug combination were reduced by half compared with the size of tumours in mice receiving treatment with cyclophosphamide alone, and the average time to tumour recurrence more than doubled. Again, these results were confirmed by inhibiting autophagy with RNA interference instead of chloroquine. Additional treatment with chloroquine had no further benefit in this setting.

This study provides evidence that autophagy can serve as an adaptive mechanism facilitating tumour cell survival and resistance to therapy-induced apoptosis, and that inhibitors of autophagy enhance the efficacy of therapeutic strategies designed to induce tumour cell apoptosis. Further investigation is

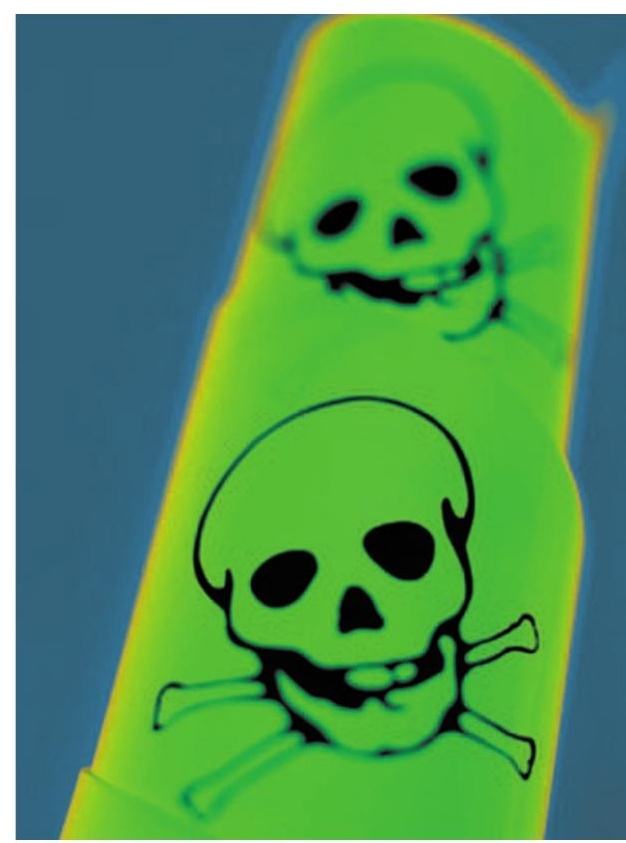

required to understand the contribution of other oncogenes and tumour suppressor genes in determining the combined outcome of apoptosis induction and autophagy inhibition. Encouragingly, autophagy has also recently been shown to be a survival pathway in AKT-driven tumours. Chloroquine and its derivatives merit further investigation as a combination therapy with systemic chemotherapy and/or radiation and in combination with recent strategies aimed at restoring p53 function in human cancer cells.

Alexandra Flemming

ORIGINAL RESEARCH PAPER Amaravadi, R. K. et al. Autophagy inhibition enhances therapyinduced apoptosis in a Myc-induced model of lymphoma. J. Clin. Invest. 117, 326-336 (2007) FURTHER READING Degenhardt, K. et al. Autophagy promotes tumor cell survival and restricts necrosis, inflammation, and tumorigenesis. Cancer Cell 10, 51-64 (2006) 